CASE REPORT

\title{
Combined fibrolamellar carcinoma and cholangiocarcinoma exhibiting biphenotypic antigen expression: a case report
}

\author{
K Tanaka, T Honna, Y Kitano, T Kuroda, K Tanaka, N Morikawa, H Matsuda, N Kawashima, \\ K Matsuoka, J Miyauchi
}

J Clin Pathol 2005;58:884-887. doi: 10.1136/icp.2004.021956

Fibrolamellar carcinoma (FLC), a variant of hepatocellular carcinoma $(\mathrm{HCC})$, very rarely occurs in association with cholangiocarcinoma (CC). This report describes the first case of FLC coexisting with CC (FLC-CC) from Japan. Although the major part of the tumour located in the right lobe of the liver showed the typical features of FLC, CC was admixed with the FLC, not only in the primary hepatic tumour, but also in the lymph node metastases. Immunohistochemical analysis revealed that, although carcinoembryonic antigen (CEA), which can be detected with monoclonal antibodies in the cytoplasm and the cell surface of CC cells but not HCC cells, was expressed in only the $\mathrm{CC}$ cells in the primary tumour, it was expressed extensively in the cytoplasm of both CC and FLC cells in the metastatic and recurrent tumours. Furthermore, Hep Par 1, a hepatocyte specific antigen, was also expressed in both the FLC and CC cells. These findings suggest that, in this case, both FLC and CC were possibly derived from the same cancer stem cell with the capacity to differentiate into both hepatocytes and bile duct epithelium, and that both the cellular components, therefore, exhibited biphenotypic antigen expression.
$\mathrm{F}$ ibrolamellar carcinoma (FLC) is a rare variant of hepatocellular carcinoma (HCC), first described by Edmondson ${ }^{1}$ in 1956, and its clinicopathological features have been reviewed by many others since..$^{2-5}$ FLC usually occurs in the liver in young patients, in the absence of underlying hepatitis or cirrhosis, and the prognosis is better than that of HCC. FLC is encountered at a much lower frequency than HCC. To date, only about 200 cases have been reported in the literature. FLC is characterised histopathologically by cords of malignant polygonal hepatocytes with eosinophilic cytoplasm surrounded by an abundant fibrous stroma arranged in lamellar bands. One other characteristic feature of FLC, seen in about half the reported cases, is the presence of sharply demarcated, pale staining cytoplasmic bodies called pale bodies. FLC is very rarely admixed with cholangiocarcinoma (CC), as in the case of HCC. ${ }^{6-8}$ To date, only nine cases of FLC coexisting with CC (FLC-CC) have been reported in the literature. ${ }^{68}$ Here, we report a case of FLC-CC of the liver, which, to our knowledge, is the first case of this type of tumour in Japan. The histological features of FLC-CC were also present in the lymph node metastases, in which intense cytoplasmic expression of carcinoembryonic antigen (CEA) was seen. These findings might provide new insight into the histogenesis of this rare tumour.

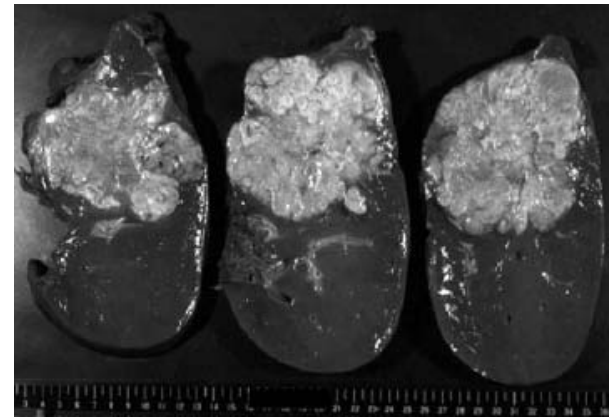

Figure 1 Gross appearance of the hepatic tumour. The cut surface of the tumour is solid, whitish yellow in colour, and exhibits scattered foci of necrosis.

"Fibrolamellar carcinoma usually occurs in the liver in young patients, in the absence of underlying hepatitis or cirrhosis, and the prognosis is better than that of hepatocellular carcinoma"

\section{CASE REPORT}

A 13 year old Japanese boy was admitted to our hospital with a two month history of right sided abdominal pain, weight loss, evening rise of body temperature, and general fatigue. He had no history of medical illness. Physical examination revealed a tender palpable left lobe of the liver. Laboratory data revealed severe anaemia (haemoglobin, $75 \mathrm{~g} / \mathrm{litre}$ ) and mildly raised CEA concentration $(24 \mathrm{ng} / \mathrm{ml})$. The serum $\alpha$ fetoprotein (AFP) and CA19-9 values were within the normal ranges, and both hepatitis $\mathrm{B}$ surface antigen and hepatitis $\mathrm{C}$ virus antibody were negative.

Computed tomography (CT) of the abdomen revealed a heterogeneous, low density tumour mass with small foci of calcification located in segments V and VIII of the liver; the hepatic hilar lymph nodes were also enlarged. Based on the CT images, age of the patient, clinical history, and laboratory data, a clinical diagnosis of FLC was made. There was no evidence of distant metastasis.

Because of the large tumour mass, chemotherapy was initiated with the following regimen: cisplatin, $80 \mathrm{mg} / \mathrm{m}^{2}$ and THP-adriamycin, $60 \mathrm{mg} / \mathrm{m}^{2}$, followed by transcatheter arterial embolisation (Lipiodol, $15 \mathrm{ml}$; THP-adriamycin, $30 \mathrm{mg}$ ). Because there was no satisfactory response, right hepatic

Abbreviations: AFP, $\alpha$ fetoprotein; CC, cholangiocarcinoma; CEA, carcinoembryonic antigen; $\mathrm{CT}$, computed tomography; EMA, epithelial membrane antigen; FLC, fibrolamellar carcinoma; $\mathrm{HCC}$, hepatocellular carcinoma; Hep Par 1, hepatocyte paraffin 1 

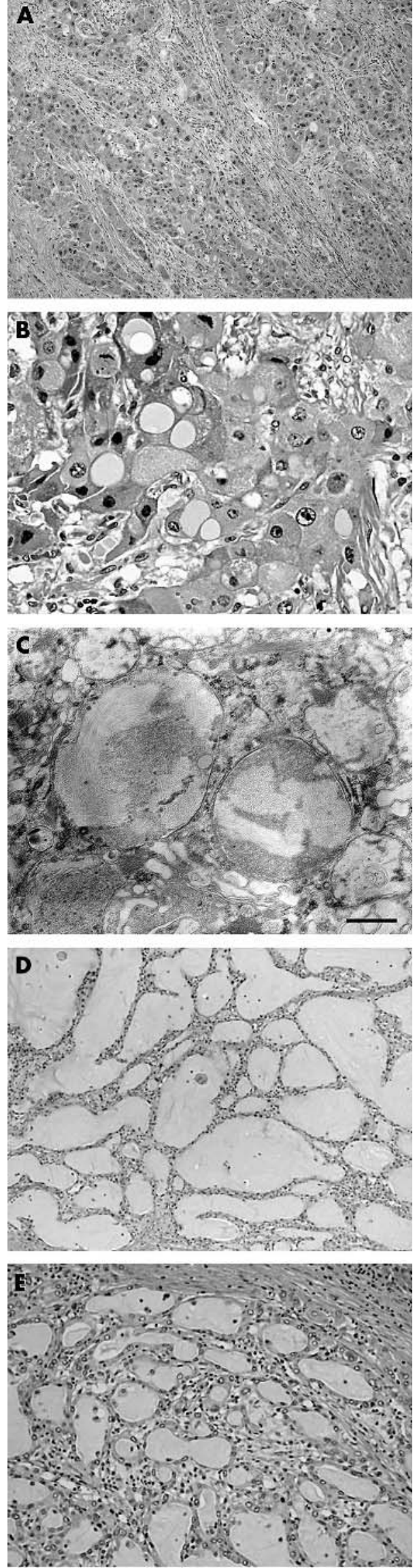

lobectomy was performed, with local lymph node dissection. The resected tumour was histopathologically diagnosed to be FLC-CC with tumour metastasis in the lymph nodes of the hepatic hilum. The serum CEA values returned to within the normal range after surgery.

Two years later, abdominal CT revealed a tumour mass, $5 \mathrm{~cm}$ in diameter, located at the hepatic hilum. The serum CEA concentration was again raised at $65.5 \mathrm{ng} / \mathrm{ml}$. The recurrent tumour mass was surgically resected after confirming the absence of distant metastasis by CT. At surgery, the tumour was found to be strongly adherent to the liver, duodenum, and the wall of the inferior vena cava. The common bile duct was entrapped within the tumour mass. The recurrent tumour was histologically confirmed to be FLCCC. Because microscopic invasion of the duodenal wall by the tumour was detected, three cycles of chemotherapy (cyclophosphamide, $1200 \mathrm{mg} / \mathrm{m}^{2}$; etoposide, $500 \mathrm{mg} / \mathrm{m}^{2}$; THP-adriamycin, $40 \mathrm{mg} / \mathrm{m}^{2}$; cisplatin, $90 \mathrm{mg} / \mathrm{m}^{2}$ ) were administered before the patient was discharged from hospital. At the time of writing, there is no evidence of local recurrence or metastasis, and the serum CEA value remains within the normal range.

\section{PATHOLOGY}

\section{Gross and histological findings}

The right hemihepatectomy specimen, $1630 \mathrm{~g}$ in weight, contained a large tumour, almost reaching the liver capsule. The tumour measured $12 \times 14 \times 8 \mathrm{~cm}$ in size and its cut surface was homogeneously whitish yellow in colour, with scattered foci of haemorrhage and necrosis (fig l). Histologically, the tumour consisted of large, hepatocyte-like neoplastic cells with eosinophilic cytoplasm, forming trabecular structures embedded in an abundant fibrous stroma arranged in lamellar bands (fig 2A). Sharply demarcated, round, pale staining structures, referred to as "pale bodies", were present in the cytoplasm of many tumour cells (fig 2B). Electron microscopic examination revealed that the pale bodies consisted of filamentous material packed densely into membrane bound spherical structures (fig 2C). These histological findings were consistent with the diagnosis of FLC. In a small part of the tumour, thick trabecular tumour nests with a sinusoidal vascular stroma, a characteristic pattern of ordinary HCC, were also present. In addition, irregular glandular structures of variable shape and size in a fibrous stroma rather than in a sinusoidal vascular stroma were seen focally within the tumour (fig 2D, E), despite a lack of evidence on gross examination. The lumens of these glandular structures were filled with mucinous material, which stained intensely with mucicarmine and Alcian blue, confirming that it was epithelial mucin produced by the tumour cells. Because HCC does not produce epithelial mucin and its presence indicates either CC or adenocarcinoma, ${ }^{6-9}$ the tumour was diagnosed as FLC-CC in accordance with the World Health Organisation criteria. ${ }^{10}$ At the marginal part of the primary tumour, the CC component was adjacent to the FLC component with a narrow transitional zone in between, but inside the tumour, the CC elements were intermingled with the FLC elements (fig 3). Tumour metastasis was

Figure 2 Histology of the tumour. (A) In fibrolamellar carcinoma, the tumour nests, consisting of large, eosinophilic neoplastic hepatocytes, are embedded in a fibrous stroma arranged in a lamellar fashion.

(B) Some of the tumour cells contain sharply demarcated, pale staining, round structures (pale bodies) in their cytoplasm. (C) Electron microscopic observation of the pale bodies reveals bundles of fine fibrillar material present in membrane bound structures. The scale bar indicates $500 \mathrm{~nm}$. (D) The cholangiocarcinoma (CC) tumour consists of irregular glandular structures surrounded by a fibrous stroma. (E) Higher magnification of an area of CC. The glandular lumens are filled with mucin. 


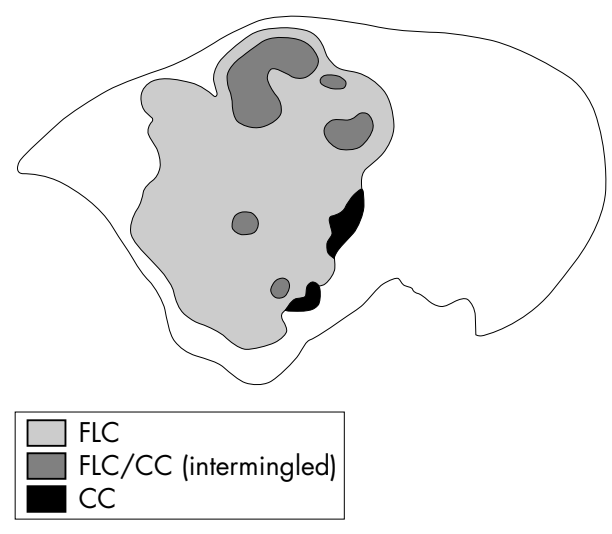

Figure 3 Distribution of cholangiocarcinoma (CC) in the fibrolamellar carcinoma (FLC) (frontal section).

present in an enlarged lymph node measuring $4 \times 3 \times 2.5 \mathrm{~cm}$ in size, detected near the hepatoduodenal ligament, which was resected during the first operation. Both the FLC and CC elements were also found to be intermingled with each other in the metastatic tumours. The recurrent tumour, resected during the second operation, which occurred as a solid mass and invaded the hepatic hilum and metastasised to the lymph nodes, also showed an admixture of FLC and CC elements.

\section{Immunohistochemistry}

Indirect immunohistochemistry was performed for CEA, hepatocyte Par l (Hep Par 1), cytokeratin, fibrinogen, $\alpha_{1}$ antitrypsin, IgG, albumin, epithelial membrane antigen (EMA), and AFP, using formalin fixed, paraffin wax embedded tissue sections and the standardised streptavidin-biotin peroxidase complex method (DAKO-LSAB; Dako Japan, Kyoto, Japan), with 3,3'-diaminobenzidine as the chromogen. All the antibodies were purchased from Dako Japan. The mouse monoclonal antibodies against CEA, Hep Par 1, and EMA were II-7, OCH1E5, and E29, respectively, and the antibodies against all the other antigens (cytokeratin, fibrinogen, $\alpha_{1}$ antitrypsin, IgG, albumin, and AFP) were rabbit polyclonal antibodies. The antibodies were used at the dilutions recommended by the manufacturer. Nuclear counterstaining was performed with haematoxylin or methyl green.

Immunohistochemistry revealed that the tumour cells of the FLC component were only focally and weakly positive for cytokeratin and EMA, whereas those of the CC components exhibited intense staining for both of these antigens. Both the FLC and CC components showed positive staining of variable intensity for fibrinogen, $\alpha_{1}$ antitrypsin, IgG, and albumin. Positive staining for CEA was also found, although focally, in the cytoplasm of the CC, but not the FLC, cells in the primary hepatic tumour (fig 4A). Interestingly, however, extensive and intense cytoplasmic staining for CEA was found in both the FLC and CC cells over wide areas in the lymph node metastases resected during the first operation and in the recurrent tumours (fig 4B). Both the FLC and CC cells also showed diffuse and intensely positive cytoplasmic staining for Hep Par 1 (fig 4C). All of the tumour cells showed consistently negative staining for AFP.

\section{DISCUSSION}

It has been reported that hepatic tumours with the histological features of FLC often contain areas of HCC as well, ${ }^{311} 12$ but only rarely, if ever, do they contain CC components. Although Goodman and colleagues ${ }^{6}$ reported that, of 24 cases of HCC coexisting with CC, eight cases
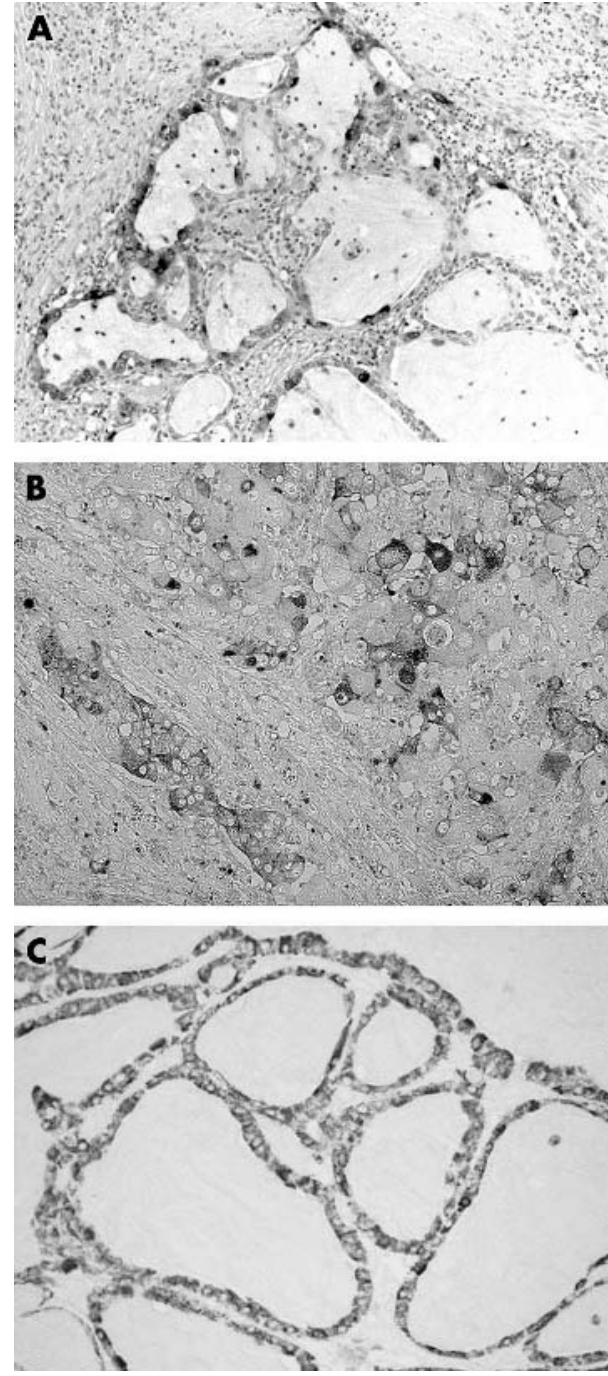

Figure 4 Immunohistochemistry of fibrolamellar carcinomacholangiocarcinoma (FLC-CC). (A) The glandular epithelium of the CC in the primary hepatic tumour shows positive cytoplasmic staining for carcinoembryonic antigen (CEA). (B) The FLC cells in the lymph node metastases show cytoplasmic staining for CEA. (C) The glandular epithelium of the CC in the primary tumour shows intense, diffuse positive staining for Hep Par 1. Nuclear counterstaining was performed with haematoxylin (A) or methyl green (B and $C)$.

showed the features of FLC-CC, only one patient with FLCCC has been reported subsequently in the literature. ${ }^{8}$ Therefore, FLC-CC is a very rare tumour and our patient is, to our knowledge, the first case of FLC-CC reported from Japan.

In our present case, although a narrow transitional zone existed between the CC and FLC elements at the marginal zone of the tumour, the two components were found to be admixed with each other in the major part of the tumour, as well as in the metastatic and recurrent tumours. Combined HCC and CC (HCC-CC) tumours are classified into two categories, the first in which HCC and CC occur independently, called "collision tumours", and the second in which both HCC and CC are intermingled with each other, and are possibly derived from a single tumour cell with bipotential differentiation capability. In this second type of tumour, both bile and mucous are simultaneously present and the tumour cells exhibit dual immunoreactivity for both the cell type specific markers. ${ }^{13}$ The admixture of FLC and CC components in our case suggests that the tumour belonged to the second 


\section{Take home messages}

- This report describes a very rare case of combined fibrolamellar and cholangiocarcinoma, in which both components were intermingled not only in the primary tumour but also in the metastatic lesions, and both the tumour components expressed carcinoembryonic antigen and Hep Par 1 in the cytoplasm

- Combined fibrolamellar and cholangiocarcinoma in this case may be derived from the same cancer stem cell with the potential to differentiate into both hepatocytes and bile duct epithelium, and which can therefore exhibit biphenotypic antigen expression

category derived from a single malignant tumour stem cell possessing the potential to differentiate into both FLC and $\mathrm{CC}$, and that the lymph node metastasis was also probably derived from such a tumour stem cell. Goodman et al further divided the second category of HCC-CC into two groups ${ }^{6}$; namely, transitional tumours and fibrolamellar tumours. Our case showed the definite histological features of FLC intermingled with mucin producing CC components within the tumour, which is consistent with a fibrolamellar tumour.

"The coexistence of cholangiocarcinoma and fibrolamellar carcinoma appears to be associated with a more aggressive clinical behaviour of the tumour"

All of the nine cases of FLC-CC described by Goodman and colleagues $^{6}$ and $\mathrm{Ng}$ and colleagues ${ }^{8}$ exhibited mucin production, the hallmark of CC, and six of them were also immunohistochemically positive for CEA. $^{6}$ It has been reported that the expression of CEA can be detected on the surface of bile canaliculi in HCC when polyclonal antibodies are used for the staining, but not when monoclonal antibodies are used, ${ }^{9}{ }^{14-16}$ and that CC exhibits both cytoplasmic and cell surface staining for CEA. ${ }^{14}$ In our patient, although only the CC component, and not the FLC component, showed positive staining for CEA using the monoclonal antibody in the primary hepatic tumour, both the FLC and CC components in the lymph node metastases and recurrent tumours showed positive cytoplasmic staining for CEA, indicating that FLC can exhibit an antigen expression pattern similar to CC under certain circumstances. In contrast, positive staining for Hep Par 1, which has been shown to be highly specific and sensitive for benign and malignant hepatocytes and is usually not seen in CC, ${ }^{17}{ }^{18}$ was found in both the FLC and CC components in all the tumours in our patient. These findings indicate that both the FLC and CC components in our patient possessed intermediate cellular characteristics between the two cell types-namely, hepatocytes and bile duct epithelium-with the potential to exhibit the antigen expression pattern of both cell types. The characteristic fibrous stroma seen in FLC might also be a reflection of the phenotypic expression of CC. These features of FLC-CC also appear to be consistent with the notion that FLC-CC might be derived from a cancer stem cell with the potential to differentiate into both of these two cell lineages.

According to the data published in the literature, the average survival period of patients with FLC is 25-126 months, 23561920 whereas that of patients with FLC-CC is only 14 months. ${ }^{6}$ Thus, the prognosis of patients with FLC-CC is much worse than that of patients with FLC alone, so that the coexistence of CC and FLC appears to be associated with a more aggressive clinical behaviour of the tumour. Because
CEA expression in, not only the CC component, but also the FLC component, was more extensive and more intense in the recurrent and metastatic tumours than in the primary tumour, CEA expression might also be associated with the aggressive biological characteristics of FLC.

\section{Authors' affiliations}

K Tanaka, T Honna, Y Kitano, T Kuroda, K Tanaka, N Morikawa,

H Matsuda, N Kawashima, Department of Surgery, National Centre for Child Health and Development, 2-10-1 Okura, Setagaya-ku, Tokyo

157-8535, Japan

K Matsuoka, J Miyauchi, Department of Clinical Laboratory, National Centre for Child Health and Development

Correspondence to: Professor J Miyauchi, Department of Pathology and Laboratory Medicine, Tokyo Dental College Ichikawa General Hospital, 5-11-13 Sugano, Ichikawa, Chiba-ken 272-8513, Japan; jmiyauchi@ tdc.ac.jp

Accepted for publication 7 February 2005

\section{REFERENCES}

1 Edmondson HA. Differential diagnosis of tumors and tumor-like lesions of liver in infancy and childhood. Am J Dis Child 1956;91:168-86.

2 Craig JR, Peters RL, Edmondson HA, et al. Fibrolamellar carcinoma of the liver: a tumor of adolescents and young adults with distinctive clinicopathologic features. Cancer 1980;46:372-9.

3 Berman MM, Libbey NP, Foster JH. Hepatocellular carcinoma, polygonal cell type with fibrous stroma: an atypical variant with a favorable prognosis. Cancer 1980;46:1448-55.

4 Farhi DC, Shikes RH, Murari PJ, et al. Hepatocellular carcinoma in young people. Cancer 1983;52:1516-25.

5 Berman MA, Burnham JA, Sheahan DG. Fibrolamellar carcinoma of the liver: an immunohistochemical study of nineteen cases and a review of the literature. Hum Pathol 1988;19:784-94.

6 Goodman ZD, Ishak KG, Langloss JM, et al. Combined hepatocellularcholangiocarcinoma: a histologic and immunohistochemical study. Cancer 1985;55:124-35.

7 Maeda T, Adachi E, Kajiyama K, et al. Combined hepatocellularcholangiocarcinoma: proposed criteria according to cytokeratin expression and analysis of clinicopathologic features. Hum Pathol 1995;26:956-64.

$8 \mathrm{Ng}$ IO, Shek TW, Nicholls J, et al. Combined hepatocellularcholangiocarcinoma: a clinicopathological study. J Gastroenterol Hepatol 1998; 13:34-40.

9 Anthony PP. Tumors of the hepatobiliary system. In: Fletcher CDM, ed. Diagnostic histopathology of tumors. London: Churchill Livingsotone, 2000:425.

10 Hirohashi S, Ishak KG, Kojiro $M$, et al. Hepatocellular carcinoma. In: Hamilton SR, Aaltonen LA, eds. WHO classification: tumors of the digestive system. Lyon: IARC Press, 2000:159-81.

11 Seitz G, Zimmermann A, Friess H, et al. Adult-type hepatocellular carcinoma in the center of a fibrolamellar hepatocellular carcinoma. Hum Pathol 2002;33:765-9.

12 Yamamoto H, Watanabe K, Nagata M, et al. Transformation of fibrolamellar carcinoma to common hepatocellular carcinoma in the recurrent lesions of the rectum and the residual liver: a case report. Jpn J Clin Oncol 1999;29:445-7.

13 Haratake J, Hashimoto H. An immunohistochemical analysis of 13 cases with combined hepatocellular and cholangiocellular carcinoma. Liver 1995; 15:9-15.

14 Porcell Al, De Young BR, Proca DM, et al. Immunohistochemical analysis of hepatocellular and adenocarcinoma in the liver: MOC31 compares favorably with other putative markers. Mod Pathol 2000;13:773-8.

15 Tsuji M, Kashihara T, Terada N, et al. An immunohistochemical study of hepatic atypical adenomatous hyperplasia, hepatocellular carcinoma, and cholangiocarcinoma with $\alpha$-fetoprotein, carcinoembryonic antigen, CA19-9, epithelial membrane antigen, and cytokeratin 18 and 19. Pathol Int 1999:49:310-17.

16 Hirohashi S, Shimosato Y, Ino Y, et al. Distrubution of alpha-fetoprotein and immunoreactive carcinoembryonic antigen in human hepatocellular carcinoma and hepatoblastoma. Jpn J Clin Oncol 1983;13:37-43.

17 Lau SK, Prakash S, Gellar SA, et al. Comparative immunohistochemical profile of hepatocellular carcinoma, cholangiocarcinoma, and metastatic adenocarcinoma. Hum Pathol 2002;33:1175-81.

18 Chu PG, Ishizawa S, Wu E, et al. Hepatocyte antigen as a marker of hepatocellular carcinoma: an immunohistochemical comparison to carcinoembryonic antigen, CD10, and alpha-fetoprotein. Am J Surg Pathol 2002;26:978-88.

19 Epstein BE, Pajak TF, Haulk TL. Metastatic nonresectable fibrolamellar hepatoma. Am J Clin Oncol 1999;22:22-8.

20 Pinna AD, Iwatsuki S, Lee RG, et al. Treatment of fibrolamellar hepatoma with subtotal hepatectomy or transplantation. Hepatology 1997;26:877-83. 